\section{BRAZIULIAN JOURNAL \\ OF MEDICAL AND BIOLOGICAL RESF.ARCH}

www.bjournal.com.br
ISSN 0100-879X

Volume 42 (12) 1119-1247 December 2009

BIOMEDICAL SCIENCES

AND

CLINICAL INVESTIGATION

Braz J Med Biol Res, December 2009, Volume 42(12) 1167-1172

Fish oil ingestion reduces the number of aberrant crypt foci and adenoma in 1,2-dimethylhydrazine-induced colon cancer in rats

A.P.B. Moreira, C.M. Sabarense, C.M.G.C. Dias, W. Lunz, A.J. Natali, M.B.A. Glória and M.C.G. Peluzio

The Brazilian Journal of Medical and Biological Research is partially financed by
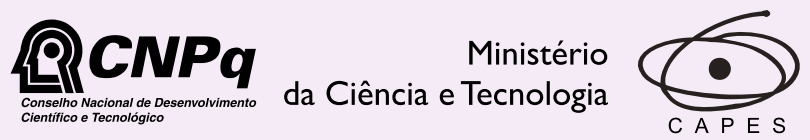

Ministério da Educação

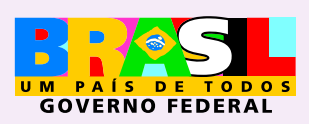

Institutional Sponsors 


\title{
Fish oil ingestion reduces the number of aberrant crypt foci and adenoma in 1,2-dimethylhydrazine-induced colon cancer in rats
}

\author{
A.P.B. Moreira ${ }^{1}$, C.M. Sabarense ${ }^{1}$, C.M.G.C. Dias ${ }^{2}$, W. Lunz ${ }^{1}$, A.J. Natali ${ }^{3}$, \\ M.B.A. Glória ${ }^{4}$ and M.C.G. Peluzio ${ }^{1}$ \\ ${ }^{1}$ Departamento de Nutrição e Saúde, ${ }^{2}$ Departamento de Biologia Animal, and \\ ${ }^{3}$ Departamento de Educação Física, Universidade Federal de Viçosa, Viçosa, MG, Brasil \\ ${ }^{4}$ LbqA, Faculdade de Farmácia, Universidade Federal de Minas Gerais, Belo Horizonte, MG, Brasil
}

\begin{abstract}
We determined the effect of fish oil (FO) ingestion on colonic carcinogenesis in rats. Male Wistar rats received 4 subcutaneous injections (40 mg/kg body weight each) of 1,2-dimethylhydrazine (DMH) at 3-day intervals and were fed a diet containing 18\% by weight FO $(\mathrm{N}=10)$ or soybean oil $(\mathrm{SO}, \mathrm{N}=10)$ for 36 weeks. At sacrifice, the colon was removed, aberrant crypt foci were counted and the fatty acid profile was determined. Intestinal tumors were removed and classified as adenoma or carcinoma. Liver and feces were collected and analyzed for fatty acid profile. FO reduced the mean ( \pm SEM) number of aberrant crypt foci compared to SO (113.55 \pm 6.97 vs $214.60 \pm 18.61$; P < 0.05) and the incidence of adenoma (FO: $20 \%$ vs SO: $100 \%)$, but carcinoma occurred equally in FO and SO rats (2 animals per group). The polyunsaturated fatty acid (PUFA) profile of the colon was affected by diet (P < 0.05): total $\omega-3$ (FO: $8.18 \pm 0.97$ vs SO: $1.71 \pm 0.54 \%$ ) and total $\omega-6$ (FO: $3.83 \pm 0.59$ vs SO: $10.43 \pm$ $1.28 \%$ ). The same occurred in the liver ( $P<0.05$ ): total $\omega-3$ (FO: $34.41 \pm 2.6$ vs SO: $6.46 \pm 0.59 \%$ ) and total $\omega-6$ (FO: $8.73 \pm$ 1.37 vs SO: $42.12 \pm 2.33 \%$ ). The PUFA profile of the feces and liver polyamine levels did not differ between groups (P > 0.05$)$. In conclusion, our findings indicate that chronic FO ingestion protected against the $\mathrm{DMH}$-induced preneoplastic colon lesions and adenoma development, but not against carcinoma in rats.
\end{abstract}

Key words: Colonic aberrant crypt foci; Colorectal cancer prevention; Colorectal tumor; Omega-3 fatty acids; Polyamines

\section{Introduction}

Colorectal cancer is the second leading cause of cancer death and the third most common type of cancer in both men and women in Western countries (1) and it is an increasing cause of death in Brazil (2). The process of carcinogenesis can be divided into three major stages: initiation, promotion and progression. Aberrant crypt foci (ACF) are considered to be putative preneoplastic colon lesions that may be early indicators of colon carcinogenesis $(3,4)$. ACF and tumors can be chemically induced by administering chemical carcinogens to experimental animals, among them, 1,2-dimethylhydrazine (DMH) or azoxymethane $(5,6)$. $\mathrm{DMH}$ is one of the agents most frequently used in experimental models of colon carcinogenesis in rodents $(6,7)$.

The occurrence of colorectal cancer is strongly correlated with nutritional factors $(8,9)$ and therefore dietary recommendations have been established to encourage people to change their habits in order to reduce the risk of colon cancer, the burden of personal suffering and the costs of treatment (10). However, the exact nature and extent of the relationship of colorectal cancer with specific nutrients is not understood (11).

Fat seems to be one of the most important determinants of colorectal cancer. Studies indicate that specific fatty acids can modulate the development of colorectal cancer $(12,13)$. For example, $\omega-3$ polyunsaturated fatty acids (PUFAs) have been shown to inhibit the in vitro growth of several human malignant cell lines (14). Fish oil is rich in $\omega$-3 PUFAs with a predominance of eicosapentaenoic acid and docosahexaenoic acid. It has been reported that fish oil may induce apoptosis and cell differentiation and reduce

Correspondence: M.C.G. Peluzio, Departamento de Nutrição e Saúde, Universidade Federal de Viçosa, Av. P.H. Rolfs, s/n, 36570-000 Viçosa, MG, Brasil. Fax: +55-31-3899-2541. E-mail: mpeluzio@ufv.br

Received October 1, 2008. Accepted November 3, 2009. Available online November 23, 2009. Published December 4, 2009. 
cell proliferation $(11,15)$. Since a diet rich in $\omega-3$ PUFAs is desirable for the prevention of colorectal cancer, it would be important to determine if fish oil would have protective effects on chemically induced colorectal cancer.

While diets rich in $\omega$-3 PUFAs can attenuate the development of colorectal cancer $(10,16)$, diets rich in $\omega-6$ PUFAs (e.g., linoleic acid) may contribute to the development of cancer (10). This is a matter of concern since Western diets usually contain high levels of $\omega-6$ PUFAs. The fatty acid composition of tissues reflects the type of dietary lipid (17). The accumulation of $\omega$-3 PUFAs in the cell membrane may act at different levels, including membrane fluidity, receptor binding, signal transduction, and enzyme activity (e.g., ornithine decarboxylase, ODC) $(11,18)$.

ODC is a key rate-limiting enzyme in polyamine biosynthesis (e.g., putrescine, spermidine, and spermine). High ODC activity and polyamine levels were found in human colorectal neoplasia (18). The bioactive amines constitute a group of cell components that are important in the regulation of cell proliferation and cell differentiation. There is evidence suggesting a role for polyamines in programmed cell death (19). The importance of the polyamines for cell function is reflected by a strict regulatory control of their intracellular levels (20). Depletion of polyamines leads to changes in the expression of numerous growth-related genes (19). However, the molecular mechanisms by which the expression of these genes is controlled are unknown (19).

Therefore, the aim of the present study was to investigate the effects of fish oil ingestion on colonic carcinogenesis in rats treated with $\mathrm{DMH}$.

\section{Material and Methods}

\section{Animals}

Eleven-week-old male Wistar rats (average weight of $248.5 \pm 5.2 \mathrm{~g}$ ) provided by the Central Animal House at the Federal University of Viçosa, MG, Brazil, were kept in collective polyethylene cages ( 3 to 4 animals per cage) in a temperature-controlled room $\left(23.7 \pm 1.6^{\circ} \mathrm{C}\right.$ and 70.2 $\pm 7.5 \%$ relative humidity) with a 12 -h dark/light cycle. All experimentation was conducted in accordance with the Ethical Principles in Animal Experimentation adopted by the Declaration of Helsinki (2000; http://www.wma.net) and by the Brazilian College on Animal Experimentation (COBEA; www.cobea.org.br).

\section{Experimental design}

Animals were randomly assigned to two groups of 10 animals each as follows: fish oil (experimental) and soybean oil (control). During the first 2 weeks of the experiment, all animals were given 4 subcutaneous injections of $40 \mathrm{mg} / \mathrm{kg}$ body weight DMH (Sigma, USA), two injections in the 1st week, and two in the 2nd week, on nonconsecutive days (7). DMH was prepared immediately before use, dissolved in $0.9 \% \mathrm{NaCl}$ containing $1.5 \%$ EDTA as vehicle at a final concentration of $10 \mathrm{mg} / \mathrm{mL}$, and final $\mathrm{pH}$ was adjusted to 6.5 . Food and water were provided ad libitum and the animals were sacrificed after an additional 36 weeks. Animal weight was monitored weekly and food intake daily throughout the experiment. Food intake of 3 rats/cage was determined and then the average diet consumption/rat was calculated.

\section{Diets}

The composition of the diets was based on the American Institute of Nutrition (21), following the AIN-93M protocol recommended for the maintenance of adult animals. Both the fish oil and soybean oil diets contained $18 \%$ by weight of lipids, simulating Western diets: $31.47 \%$ cornstarch, $15.5 \%$ dextrinized cornstarch, $10 \%$ sucrose, $15.1 \%$ casein, $18 \%$ fish oil or soybean oil, $5 \%$ cellulose, $3.5 \%$ mineral mix, $1 \%$ vitamin mix, $0.18 \%$ L-cystine, and $0.25 \%$ choline bitartrate.

\section{Sample collection}

After sacrifice, the large intestine was removed, opened longitudinally, washed gently, and fixed flat on a styrofoam board in $10 \%$ buffered formaldehyde for $48 \mathrm{~h}$ for ACF analysis. One fragment of the large intestine was collected, frozen in liquid nitrogen, and kept at $-80^{\circ} \mathrm{C}$ until the analysis of fatty acid profile. Intestinal tumors were removed and fixed in $10 \%$ buffered formaldehyde for further analysis. The liver was removed, frozen in liquid nitrogen and kept at $-80^{\circ} \mathrm{C}$ until the analyses of fatty acids and polyamines. All animal feces were collected from each cage during the 15 days that preceded euthanasia and kept at $-20^{\circ} \mathrm{C}$ until the analysis of fatty acids.

\section{Fatty acid analysis}

The lipids from the oils used in the diets, from tissues (colon - portion between the middle and distal segments and liver) and from feces were extracted with 2:1 chloroform:methanol (22) prior to analysis. The fatty acids were esterified (23) and tridecanoic acid - C13:0 (SigmaAldrich, USA) was used as an internal standard. The fatty acid methyl esters were determined by gas chromatography (Shimadzu, Japan). The fatty acid methyl esters were identified by comparing the retention times of the samples with reference to standards (Sigma-Aldrich) and quantified by the method of Satchithanandam et al. (24). Fecal lipids were measured in the lipid fractions from the pool of feces obtained from each cage.

\section{Counts of aberrant crypt foci}

After $48 \mathrm{~h}$ of fixation the intestine was divided into three fragments of equal length $(\sim 8 \mathrm{~cm})$, i.e., proximal, middle and distal regions. They were stained with $0.1 \%$ methylene blue for approximately 2 min and washed in phosphate buffer. ACF were identified and counted under a light microscope (100X) by two pathologists unaware of the treatments $(4,7)$. The ACF were grouped as a function of the size of the foci: 
small ACF (aberrant crypts per focus $\leq 3, A C F \leq 3$ ), and large ACF (aberrant crypts per focus $\geq 4, A C F \geq 4$ ) (4).

\section{Tumor analysis}

Intestinal tumors were prepared for conventional histopathological analysis. After fixation, the material was dehydrated in alcohol, diaphanized in xylol and embedded in paraffin. Four-micrometer thick sections were cut, stained with hematoxylin and eosin and examined at 40X magnification under a light microscope. Tumors were analyzed by using three to four slides and classified as either adenoma or carcinoma by the method of Pozharisski (25).

\section{Polyamine analysis}

Liver spermidine, spermine and putrescine levels were determined. The amines were extracted with $5 \mathrm{~g} / 100 \mathrm{~mL}$ trichloroacetic acid by the method of Vale and Glória (26). The extract was analyzed by ion pair high performance liquid chromatography using ion pairing in a reverse phase column, post-column derivatization with o-phthalaldehyde and fluorescent detection at $340 \mathrm{~nm}$ excitation and 550 $\mathrm{nm}$ emission (26).

\section{Statistical analysis}

Data were analyzed by the Mann-Whitney test or the Student $t$-test. The Fisher exact method was used to analyze tumor incidence. Data were analyzed with the Sigma Stat for Windows software (SPSS Inc., USA) and differences were considered to be significant at $P<0.05$.

\section{Results}

The fatty acid composition of the oils used are given in Table 1. The major differences are (means $\pm \mathrm{SD}$ ): fish oil ( $\omega-3$ fatty acids: $44.63 \pm 2.24 \%$; $\omega-6$ fatty acids: $4.37 \pm$ $0.15 \%)$ and soybean oil ( $\omega-3$ fatty acid: $6.93 \pm 0.11 \%$; $\omega-6$ fatty acid: $60.75 \pm 1.79 \%)$.

Food intake did not differ significantly between the fish oil and soybean oil groups. Furthermore, animals gained weight similarly throughout the experiment irrespective of treatment (data not shown).

The total ACF number and the number of small ACF $(\leq 3)$ were significantly lower in the fish oil group compared to control (soybean oil). However, the number of large ACF $(\geq 4)$ did not differ between groups (Table 2).

The incidence of adenomas was $20 \%$ in the fish oil group (2 of 10 rats) and $100 \%$ in the soybean oil group (10 of 10 rats), whereas the incidence of carcinomas was equal in rats consuming fish oil (2 of 10 rats) or soybean oil (2 of 10 rats).

While animals receiving fish oil presented a higher percentage of total $\omega$-3 PUFAs in the colon than those receiving soybean oil, animals from the soybean oil group showed a higher percentage of total $\omega-6$ PUFAs than animals from the fish oil group (Table 3). Similarly, a higher percentage of
Table 1. Fatty acid composition of the fish and soybean oils used in the present study.

\begin{tabular}{|c|c|c|}
\hline Fatty acid & Fish oil & Soybean oil \\
\hline Linoleic acid (C18:2w-6) & $2.78 \pm 0.09$ & $60.75 \pm 1.79$ \\
\hline 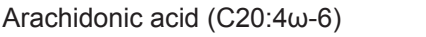 & $0.73 \pm 0.04$ & - \\
\hline Docosadienoic acid (C22:2w-6) & $0.87 \pm 0.02$ & - \\
\hline$\alpha-$ Linolenic acid (C18:3w-3) & $0.80 \pm 0.04$ & $6.93 \pm 0.11$ \\
\hline Eicosapentaenoic acid (C20:5w-3) & $24.01 \pm 1.07$ & - \\
\hline Docosahexaenoic acid (C22:6w-3) & $19.82 \pm 1.15$ & - \\
\hline Total $\omega-6$ & $4.37 \pm 0.15$ & $60.75 \pm 1.79$ \\
\hline Total $\omega-3$ & $44.63 \pm 2.24$ & $6.93 \pm 0.11$ \\
\hline
\end{tabular}

Data are reported as means \pm SD of weight percent for 3 independent analyses. $\omega-6$ or $\omega-3$ free fatty acids were determined by gas chromatography. - = not detected.

Table 2. Effects of fish oil or of soybean oil diets on aberrant crypt foci (ACF) in the large intestine of rats treated with 1,2-dimethylhydrazine.

\begin{tabular}{lcc}
\hline & Fish oil & \multicolumn{1}{c}{ Soybean oil } \\
\hline ACF $\leq 3$ & $90.55 \pm 4.74$ & $184.35 \pm 14.70^{*}$ \\
ACF $\geq 4$ & $23.00 \pm 3.21$ & $30.25 \pm 5.81$ \\
Total ACF & $113.55 \pm 6.97$ & $214.60 \pm 18.61^{*}$ \\
\hline
\end{tabular}

Data are reported as means \pm SEM for 10 rats in each group Rats received 4 subcutaneous injections of 1,2-dimethylhydrazine (40 mg/kg body weight each) twice a week on nonconsecutive days for 2 weeks and were submitted to diets containing $18 \%$ of either fish oil or soybean oil ad libitum for 36 weeks. * $\mathrm{P}<$ 0.05 compared to fish oil in diet (Mann-Whitney test).

$\omega$-3 PUFAs was observed in the liver of animals ingesting the fish oil than in the liver of animals of the soybean oil group, whereas the percentage of $\omega-6$ PUFAs was higher in the soybean oil group (Table 3). Although fish oil contains a higher percentage of eicosapentaenoic acid than docosahexaenoic acid, animals in the fish oil group presented a higher percentage of docosahexaenoic acid than animals of the soybean group in both colon and liver (Table 3).

The profile of fatty acids in the animals' feces was different from that accumulated in the liver and colon, with a prevalence of saturated fatty acids. The fish oil group excreted a significantly higher percentage of myristic acid (1.03 \pm 0.16 vs $0.23 \pm 0.01 \%)$, pentadecanoic acid $(0.35 \pm$ 0.01 vs $0.21 \pm 0.05 \%)$, palmitic acid (7.50 \pm 1.03 vs 7.20 $\pm 0.55 \%)$, and heptadecanoic acid $(0.59 \pm 0.06$ vs $0.23 \pm$ $0.01 \%$ ) compared to the soybean oil group. However, the feces of animals of the soybean oil group were significantly higher in docosanoic acid than animals of the fish oil group $(1.24 \pm 0.03$ vs $0.49 \pm 0.07 \%)$. No differences were observed between groups in total $\omega-3$ PUFAs (fish oil: $0.84 \pm 0.18 v s$ soybean oil: $0.98 \pm 0.38 \%$ ) or in total $\omega-6$ PUFAs (fish oil: 
Table 3. Effects of fish oil (FO) or soybean oil (SO) diets on the fatty acid profile of large intestine and liver of rats treated with 1,2-dimethylhydrazine.

\begin{tabular}{|c|c|c|c|c|}
\hline \multirow[t]{2}{*}{ Fatty acid (\%) } & \multicolumn{2}{|c|}{ Large intestine } & \multicolumn{2}{|c|}{ Liver } \\
\hline & FO & so & FO & so \\
\hline Myristic acid (C14:0) & $1.37 \pm 0.36$ & - & $0.54 \pm 0.17$ & - \\
\hline Pentadecanoic acid (C15:0) & $0.15 \pm 0.03$ & - & $0.12 \pm 0.01$ & - \\
\hline Palmitic acid (C16:0) & $8.06 \pm 1.20^{*}$ & $3.83 \pm 0.41$ & $10.79 \pm 0.99$ & $11.09 \pm 0.94$ \\
\hline Stearic acid (C18:0) & $4.02 \pm 0.79$ & $4.21 \pm 1.13$ & $5.99 \pm 1.07$ & $4.40 \pm 1.11$ \\
\hline Palmitoleic acid (C16:1) & $2.65 \pm 0.56$ & - & $2.56 \pm 0.92^{*}$ & $0.84 \pm 0.23$ \\
\hline Oleic acid (C18:1w-9) & $7.75 \pm 1.87^{*}$ & $4.39 \pm 0.65$ & $9.82 \pm 0.83$ & $12.28 \pm 0.88$ \\
\hline Eicosanoic acid (C20:1) & $0.32 \pm 0.10$ & - & $0.25 \pm 0.01$ & - \\
\hline Eicosadienoic acid (C20:2) & $0.46 \pm 0.07$ & - & - & - \\
\hline Linoleic acid (C18:2w-6) & $2.12 \pm 0.34^{*}$ & $5.43 \pm 1.94$ & $4.23 \pm 0.98^{*}$ & $32.99 \pm 2.87$ \\
\hline y-linolenic acid $(\mathrm{C} 18: 3 \omega-6)$ & - & $0.97 \pm 0.15$ & - & - \\
\hline Dihomo-y-linolenic acid (C20:3w-6) & - & $0.81 \pm 0.23$ & - & $0.64 \pm 0.11$ \\
\hline Arachidonic acid (C20:4w-6) & $1.71 \pm 0.55^{*}$ & $4.19 \pm 1.39$ & $3.86 \pm 0.88^{*}$ & $7.50 \pm 1.03$ \\
\hline Docosadienoic acid (C22:2w-6) & $0.64 \pm 0.14$ & - & - & - \\
\hline$\alpha$-linolenic acid (C18:3w-3) & $0.69 \pm 0.24^{*}$ & $1.99 \pm 0.29$ & - & - \\
\hline Eicosapentaenoic acid (C20:5w-3) & $2.71 \pm 0.64$ & - & $9.80 \pm 2.65$ & - \\
\hline Docosahexaenoic acid (C22:6w-3) & $5.47 \pm 0.83^{*}$ & $1.71 \pm 0.54$ & $23.91 \pm 1.69^{*}$ & $4.47 \pm 0.83$ \\
\hline Total $\omega-3$ & $8.18 \pm 0.97^{*}$ & $1.71 \pm 0.54$ & $34.41 \pm 2.6^{*}$ & $6.46 \pm 0.59$ \\
\hline Total $\omega-6$ & $3.83 \pm 0.59^{*}$ & $10.43 \pm 1.28$ & $8.73 \pm 1.37^{*}$ & $42.12 \pm 2.33$ \\
\hline
\end{tabular}

Data are reported as means \pm SD as $\%$ by weight for 10 rats in each group. Rats received 4 subcutaneous injections of 1,2-dimethylhydrazine (40 mg/kg body weight each) twice a week on nonconsecutive days for 2 weeks and were submitted to diets containing $18 \%$ of either fish oil or soybean oil ad libitum for 36 weeks. $-=$ not detected. ${ }^{*} \mathrm{P}<0.05$ compared to soybean oil-treated group of the same tissue (Student $t$-test).

$2.37 \pm 0.44$ vs soybean oil: $3.14 \pm 0.17 \%)$.

The prevalent polyamine in the liver was spermine, followed by spermidine. Putrescine, which is a precursor of polyamines, was not detected in the liver of animals in either group. There was no significant difference in the levels of spermine (fish oil: $4.08 \pm 1.86$ and soybean oil: $5.42 \pm 0.51 \mathrm{mg} / 100 \mathrm{~g}$ ) or spermidine (fish oil: $3.08 \pm 1.15$ and soybean oil: $3.15 \pm 0.50 \mathrm{mg} / 100 \mathrm{~g}$ ).

\section{Discussion}

In this study of the effect of fish oil on colon carcinogenesis, we showed that rats consuming a diet enriched in fish oil $(18 \%)$ for 36 weeks exhibited a significant reduction in $\mathrm{DMH}$-induced ACF. This reduction was due to the reduction of ACF $\leq 3$ as ACF $\geq 4$ was not affected. Since ACF is a putative preneoplastic colon lesion that may be an early indicator of colon carcinogenesis (3), these findings suggest that the ingestion of fish oil protected against the initiation of ACF development in the rat colon.

We also investigated the effect of fish oil on the incidence of tumors. Our results demonstrated that the incidence of adenomas was significantly reduced in animals receiving a diet containing fish oil, whereas the incidence of carcinomas was not affected by the type of diet consumed. On the basis of these results, it is likely that fish oil consumption may protect against the development of colonic tumors in the stage before the formation of carcinomas.

Although our study was not designed to identify mechanisms, the inhibitory effects of $\omega$-3 PUFAs on carcinogenesis have been attributed by others to cyclooxygenase- 2 downregulation, and to the decreased production of prostaglandin E2 as a result of the competition between $\omega-6$ and $\omega-3$ PUFAs for desaturases, elongases, cyclooxygenases, and lipoxygenase enzymes (10). It is likely that $\omega$-3 PUFAs have greater affinity for these enzymes than do $\omega-6$ PUFAs (15). Prostaglandin E2 itself is known to promote carcinogenesis by inhibiting apoptosis and inducing cell proliferation and angiogenesis $(27,28)$. Furthermore, eicosanoids produced from eicosapentaenoic acid are less pro-inflammatory than the arachidonic acid-derived mediators (10).

These are the first observations regarding the effects of the ingestion of fish oil for a long period on the lipid profile of large intestine, liver and feces. Our data showed that colon and liver had a fatty acid profile similar to that of the oil in the diet. The group that received fish oil presented a 
higher concentration of $\omega-3$ PUFAs in the colon and liver, whereas the group treated with soybean oil presented a higher concentration of $\omega-6$ PUFAs. These results confirm and extend data reported by Hendrickse et al. (17) and Senkal et al. (29) showing that the fatty acid composition of tissues reflects the type of dietary fat. In addition, the higher content of docosahexaenoic acid in the colon and liver of rats from the fish oil group in the present study suggests that eicosapentaenoic acid from the diet was converted to docosahexaenoic acid by the delta-6-desaturase enzyme. It should be mentioned that the levels of eicosapentaenoic acid in the diet were higher than those of docosahexaenoic acid.

We also showed that $\omega-3$ and $\omega-6$ PUFA excretion through the feces was similar for the groups studied, but was lower than in the liver and colon, suggesting a high utilization of PUFAs present in the experimental diets by the animals.

Eicosapentaenoic acid was not detected in the tissues or feces of animals, which ingested soybean oil, but docosahexaenoic acid was detected. These animals received

\section{References}

1. ACS (American Cancer Society). Cancer facts and figures, 2007. http://www.cancer.org. Accessed June 20, 2008.

2. Brasil. Ministério da Saúde. Secretaria de Atenção à Saúde. Instituto Nacional de Câncer. Coordenação de Prevenção e Vigilância: Estimativas 2008. Incidência de Câncer no Brasil. Rio de Janeiro: INCA. 2007. http://www.inca.gov.br. Accessed July 11, 2009.

3. Fenoglio-Preiser CM, Noffsinger A. Aberrant crypt foci: $A$ review. Toxicol Pathol 1999; 27: 632-642.

4. Bird RP. Observation and quantification of aberrant crypts in the murine colon treated with a colon carcinogen: preliminary findings. Cancer Lett 1987; 37: 147-151.

5. Rao CV, Hirose $Y$, Indranie C, Reddy BS. Modulation of experimental colon tumorigenesis by types and amounts of dietary fatty acids. Cancer Res 2001; 61: 1927-1933.

6. Lunz W, Peluzio MC, Dias CM, Moreira AP, Natali AJ. Longterm aerobic swimming training by rats reduces the number of aberrant crypt foci in 1,2-dimethylhydrazine-induced colon cancer. Braz J Med Biol Res 2008; 41: 1000-1004.

7. Rodrigues MA, Silva LA, Salvadori DM, de Camargo JL, Montenegro MR. Aberrant crypt foci and colon cancer: comparison between a short- and medium-term bioassay for colon carcinogenesis using dimethylhydrazine in Wistar rats. Braz J Med Biol Res 2002; 35: 351-355.

8. Navarro A, Munoz SE, Lantieri MJ, del Pilar DM, Cristaldo $\mathrm{PE}$, de Fabro SP, et al. Meat cooking habits and risk of colorectal cancer in Cordoba, Argentina. Nutrition 2004; 20: 873-877.

9. Roberts CK, Barnard RJ. Effects of exercise and diet on chronic disease. J Appl Physiol 2005; 98: 3-30.

10. Roynette CE, Calder PC, Dupertuis YM, Pichard C. n-3 polyunsaturated fatty acids and colon cancer prevention. Clin Nutr 2004; 23: 139-151. soybean oil that contains $\alpha$-linolenic acid which, by elongations and desaturations, may give rise to eicosapentaenoic acid, and hence docosahexaenoic acid.

Finally, because of the significant difference in the incidence of adenomas between our experimental groups, we investigated the levels of polyamines in the liver of these animals. Nevertheless, our results demonstrated that fish oil did not alter the content of bioactive amines in the liver of rats with $\mathrm{DMH}$-induced colon carcinogenesis.

Our data showed that the chronic consumption of fish oil protected intestinal mucosa against the $\mathrm{DMH}$-induced preneoplastic lesions and adenoma development, but not against carcinoma in rats. However, further studies are necessary to elucidate the physiological role of fish oil in colon carcinogenesis, and these findings may be useful in the quest for prevention of colon cancer.

\section{Acknowledgments}

Research supported by CAPES.

11. Llor X, Pons E, Roca A, Alvarez M, Mane J, FernandezBanares $F$, et al. The effects of fish oil, olive oil, oleic acid and linoleic acid on colorectal neoplastic processes. Clin Nutr 2003; 22: 71-79.

12. Fernandez E, Chatenoud L, La Vecchia C, Negri E, Franceschi S. Fish consumption and cancer risk. Am J Clin Nutr 1999; 70: 85-90.

13. Nkondjock A, Shatenstein B, Maisonneuve P, Ghadirian P. Assessment of risk associated with specific fatty acids and colorectal cancer among French-Canadians in Montreal: a case-control study. Int J Epidemiol 2003; 32: 200-209.

14. Dupertuis YM, Benais-Pont G, Buchegger F, Pichard C. Effect of an immunonutrient mix on human colorectal adenocarcinoma cell growth and viability. Nutrition 2007; 23 : $672-680$

15. Benais-Pont G, Dupertuis YM, Kossovsky MP, Nouet P, Allal AS, Buchegger F, et al. Omega-3 polyunsaturated fatty acids and ionizing radiation: combined cytotoxicity on human colorectal adenocarcinoma cells. Nutrition 2006; 22: 931-939.

16. Cho Y, Martinez JG, Turner ND, Taddeo SS, Davidson LA, Wang $N$, et al. Fish oil and pectin may suppress colon carcinogenesis via inhibition of the MAPK and TGF $\beta$ pathways. FASEB J 2008; 22: 885-888.

17. Hendrickse CW, Keighley MR, Neoptolemos JP. Dietary omega-3 fats reduce proliferation and tumor yields at colorectal anastomosis in rats. Gastroenterology 1995; 109: 431-439.

18. LaMuraglia GM, Lacaine F, Malt RA. High ornithine decarboxylase activity and polyamine levels in human colorectal neoplasia. Ann Surg 1986; 204: 89-93.

19. Medina MA, Urdiales JL, Rodriguez-Caso C, Ramirez FJ, Sanchez-Jimenez F. Biogenic amines and polyamines: similar biochemistry for different physiological missions and 
biomedical applications. Crit Rev Biochem Mol Biol 2003; 38: 23-59.

20. Gerner EW, Meyskens FL Jr. Combination chemoprevention for colon cancer targeting polyamine synthesis and inflammation. Clin Cancer Res 2009; 15: 758-761.

21. Reeves PG, Nielsen FH, Fahey GC Jr. AIN-93 purified diets for laboratory rodents: final report of the American Institute of Nutrition ad hoc writing committee on the reformulation of the AIN-76A rodent diet. J Nutr 1993; 123: 1939-1951.

22. Folch J, Lees M, Sloane Stanley GH. A simple method for the isolation and purification of total lipides from animal tissues. J Biol Chem 1957; 226: 497-509.

23. Hartman L, Lago RC. Rapid preparation of fatty acid methyl esters from lipids. Lab Pract 1973; 22: 475-476.

24. Satchithanandam S, Fritsche J, Rader JI. Gas chromatographic analysis of infant formulas for total fatty acids, including trans fatty acids. J AOAC Int 2002; 85: 86-94.

25. Pozharisski KM. Morphology and morphogenesis of experi- mental epithelial tumors of the intestine. J Natl Cancer Inst 1975; 54: 1115-1135.

26. Vale SR, Gloria MB. Determination of biogenic amines in cheese. J AOAC Int 1997; 80: 1006-1012.

27. Leahy KM, Ornberg RL, Wang Y, Zweifel BS, Koki AT, Masferrer JL. Cyclooxygenase-2 inhibition by celecoxib reduces proliferation and induces apoptosis in angiogenic endothelial cells in vivo. Cancer Res 2002; 62: 625-631.

28. Pai R, Nakamura T, Moon WS, Tarnawski AS. Prostaglandins promote colon cancer cell invasion; signaling by crosstalk between two distinct growth factor receptors. FASEB $J$ 2003; 17: 1640-1647.

29. Senkal M, Haaker R, Linseisen J, Wolfram G, Homann HH, Stehle P. Preoperative oral supplementation with long-chain Omega-3 fatty acids beneficially alters phospholipid fatty acid patterns in liver, gut mucosa, and tumor tissue. JPEN $J$ Parenter Enteral Nutr 2005; 29: 236-240. 